OPEN ACCESS

Edited by:

Jens Staal,

Ghent University, Belgium

Reviewed by:

Marta Berrocal-Lobo,

Universidad Politécnica de Madrid

(UPM), Spain

Kemal Kazan,

Commonwealth Scientific

and Industrial Research Organisation

(CSIRO), Australia

*Correspondence:

Fuguang $L$

aylifug@126.com

Yuxia Hou

yuxiacau@163.com

Specialty section:

This article was submitted to

Plant Microbe Interactions,

a section of the journal

Frontiers in Plant Science

Received: 27 February 2018

Accepted: 26 April 2018

Published: 24 May 2018

Citation:

Li $X$, Pei $Y$, Sun Y, Liu N, Wang P, Liu D, Ge X, Li F and Hou Y (2018)

A Cotton Cyclin-Dependent Kinase $E$

Confers Resistance to Verticillium dahliae Mediated by

Jasmonate-Responsive Pathway.

Front. Plant Sci. 9:642.

doi: 10.3389/fpls.2018.00642

\section{A Cotton Cyclin-Dependent Kinase E Confers Resistance to Verticillium dahliae Mediated by Jasmonate-Responsive Pathway}

\author{
Xiancai Li ${ }^{1}$, Yakun Pei ${ }^{1}$, Yun Sun ${ }^{1}$, Nana Liu ${ }^{1}$, Ping Wang ${ }^{1}$, Di Liu' ${ }^{1}$, Xiaoyang Ge ${ }^{2}$, \\ Fuguang $\mathrm{Li}^{2 *}$ and Yuxia Hou ${ }^{1 *}$ \\ ${ }^{1}$ College of Science, China Agricultural University, Beijing, China, ${ }^{2}$ State Key Laboratory of Cotton Biology, Institute of \\ Cotton Research, Chinese Academy of Agricultural Sciences, Anyang, China
}

Many subunits of the Mediator transcriptional co-activator complex are multifunctional proteins that regulate plant immunity in Arabidopsis. Cotton cyclin-dependent kinase $\mathrm{E}$ (GhCDKE), which is a subunit of the cotton (Gossypium hirsutum) Mediator complex, has been annotated, but the biological functions of this gene associated with regulating disease resistance have not been characterized. Here, we cloned GhCDKE from cotton and confirmed that GhCDKE belonged to the E-type CDK family in the phylogenetic tree, and, as in other eukaryotes, we found that GhCDKE interacted with C-type cyclin (GhCycC) by yeast two-hybrid and luciferase complementation imaging assays. Expression of GhCDKE in cotton was induced by Verticillium dahliae infection and MeJA treatment, and silencing of GhCDKE expression in cotton led to enhanced susceptibility to $V$. dahliae, while overexpression of GhCDKE in Arabidopsis thaliana enhanced resistance to this pathogen. Transgenic expression assay demonstrated that the transcriptional activity of GhPDF1.2 pro:LUC in GhCDKE-silenced cotton was dramatically inhibited. In addition, the expression of jasmonic acid (JA)-regulated pathogen-responsive genes was dramatically upregulated in GhCDKE-overexpressed plants after inoculation with $V$. dahliae, and the roots of GhCDKE-overexpressed A. thaliana were more susceptible to JA treatment. These results indicated that GhCDKE regulates resistance against $V$. dahliae and that this resistance is mediated by JA response pathway.

Keywords: cotton (Gossypium hirsutum), GhCDKE, Verticillium dahliae, jasmonate signal, VIGS

\section{INTRODUCTION}

Cotton is an important crop globally because of its fiber. However, yield and quality can be negatively affected by the fungus Verticillium dahliae (Fradin and Thomma, 2006). Several factors make it difficult to control this disease. First, the host range of Verticillium dahliae is broad, and the fungus can survive in the soil for many years, even in the absence of hosts (Ma et al., 1997). Moreover, studies have shown that pathogenic types of $V$. dahliae in cotton fields comprise a highly diverse community, which continuously changes from weak to strong type (Zhu et al., 2004). 
Although this disease is particularly difficult to control, efforts have been made to investigate the molecular mechanisms underlying cotton resistance to $V$. dahliae infection. Many resistance and resistance-related genes have been discovered. For example, the immune receptor $\mathrm{Ve} 1$, which is responsible for $V$. dahliae resistance, has been cloned from tomato (Kawchuk et al., 2001; de Jonge et al., 2012). Importantly, it has been shown that interfamily transfer of Ve1 into Arabidopsis thaliana confers resistance to race 1 strains of $V$. dahliae (Fradin et al., 2011). Using a virus-induced gene silencing (VIGS) approach, the signaling cascade downstream of Vel requires both EDS1 and NDR1. ACIF, MEK2, BAK1, and NRC1 also act as positive regulators of Vel (Fradin et al., 2009). In addition, sets of genes/proteins, including components in ethylene (ET)-, salicylic acid (SA)-, and jasmonic acid (JA)-mediated signaling pathways, have been shown to be functionally related to $V$. dahliae resistance (Xu et al., 2014). These studies have taken important steps toward discovering the complex disease resistance mechanisms against $V$. dahliae infection in cotton (Kawchuk et al., 2001; Fradin et al., 2009; Xu et al., 2014).

Transcription regulation of defense-related genes is important in plant-pathogen interactions. In eukaryotes, a highly conserved multiprotein complex called the Mediator complex mediates the interaction between transcription regulators and RNA polymerase II (RNAPII) (Kim et al., 1994; Koleske and Young, 1994). The core of the Mediator is composed of three discrete modules (head, middle, and tail) and a separable kinase module consisting of Mediator complex subunit 12 (MED12), MED13, cyclin-dependent kinase 8 (CDK8), and C-type cyclin (CycC; Guglielmi et al., 2004; Andrau et al., 2006). Different Mediator subunits interact with particular transcription activators to transfer various signals to the RNAPII transcription complex and results in the transcription of specific pathway genes (Donner et al., 2007). For example, the subunit MED2/MED32 regulates the expression of cold-responsive genes in Arabidopsis (Hemsley et al., 2014).

Recent studies have shown that Mediator subunits are associated with plant immunity to bacterial and fungal infection. For instance, MED25 is a positive regulator of JA-responsive gene expression, and mutation of this gene leads to increased resistance to the hemibiotroph Fusarium oxysporum and susceptibility to the necrotrophic pathogens Botrytis cinerea and Alternaria brassicicola (Kidd et al., 2009; Çevik et al., 2012; Chen et al., 2012). MED15/NRB4 functions downstream of NPR1 to regulate the SA response (Canet et al., 2012). HaRxL44, the nuclear-localized effector of Hyaloperonospora arabidopsidis (Hpa), interacts with MED19a, shifting the balance of defense transcription from the SA to the JA/ET-signaling pathway and increasing susceptibility to biotrophic pathogens by reducing SA-responsive gene expression (Caillaud et al., 2013). MED14, MED15, and MED16 have all been shown to be essential for the induction of systemic acquired resistance (Zhang et al., 2012, 2013; Wang et al., 2016). These results suggest that the Mediator complex acts as an integrative hub for regulation of specific signaling processes.

Here, we aimed to discover whether cotton cyclin-dependent kinase E (GhCDKE) plays a role in transcriptional response pathways, particularly those utilized in plant defenses against pathogen infection. To do this, we cloned GhCDKE, a subunit of the Mediator complex that interacts with $\mathrm{CycC}$. We showed that GhCDKE is required for defense against $V$. dahliae infection, and we demonstrated that GhCDKE is required for a JA-mediated defense response after inoculation with $V$. dahliae. Our results indicated that GhCDKE is a key regulator of basal resistance against $V$. dahliae.

\section{MATERIALS AND METHODS}

\section{Plant Material}

The seedlings of Zhongzhimian 2, a $V$. dahliae-tolerant breeding line of upland cotton, were cultivated under standard conditions of $26^{\circ} \mathrm{C}$ and a 16 -h photoperiod. Tobacco (Nicotiana benthamiana) plants were grown in an incubator under controlled environmental conditions $\left(22^{\circ} \mathrm{C}, 16 \mathrm{~h}\right.$ photoperiod) as described previously (Talarczyk et al., 2002). A. thaliana seeds were germinated on Murashige and Skoog (MS) medium and cultured at $22^{\circ} \mathrm{C}$ under constant illumination, and 14-days-old seedlings were transferred to soil.

\section{RNA Extraction, RT-PCR, and qRT-PCR}

Total RNA was isolated from cotton plants that were infected with Vd991 or exposed to $100 \mu \mathrm{M}$ MeJA using an RNA extraction kit (Biomed, Enzo Life Sciences, Exeter, United Kingdom). The first strand of cDNA was synthesized from $2 \mu \mathrm{g}$ of total RNA with a FASTQuant cDNA RT Kit (TIANGEN Biotech Co., Ltd) and used as template in quantitative reverse transcription polymerase chain reaction (qRT-PCR). The cotton GhUBQ gene was used as an internal control. Primers were designed as shown in Supplementary Table S1. Reactions were prepared in $20-\mu \mathrm{L}$ tubes using SYBR ${ }^{\circledR}$-Premix Ex Taq (Tli RNaseH Plus; Takara, Shiga, Japan) and amplified on an ABI 7500 thermocycler (Applied Biosystems, Foster City, CA, United States). Expression was determined by the $2^{-\Delta \Delta C T}$ method, and data were analyzed in Origin 8 (Liu et al., 2017).

\section{Disease Assays in Transgenic Arabidopsis Plants and Cotton}

To obtain GhCDKE-overexpressing Arabidopsis plants, GhCDKE was amplified using the forward (5'AAACTGCAGATGGGAGATGGCAATAACA- $3^{\prime}$ ) and reverse (5'-TAGACTAGTCATGCGTCTCGATTTTTGC-3') primers, which incorporated Pat1 and Spe1 cleavage sites, respectively. Next, the gene was cloned into vector Super-pCAMBIA1300. The plasmid was transformed into Agrobacterium tumefaciens strain GV3101. Agrobacterium-mediated transformation of A. thaliana was performed by floral dip method (Zhang et al., 2006).

The $V$. dahliae strain V991 was used for the inoculation assays. Fungal colonies were grown on potato-dextrose agar medium for 7 days and then the fungus was inoculated into Czapek medium at $25^{\circ} \mathrm{C}$ for 1 week at which time the spores were harvested. The spore suspension was adjusted to $10^{6} \mathrm{~mL}^{-1}$ with deionized water for the inoculation assays. Two-weeks-old cotton seedlings 
were removed from the soil and dip-infected into the spore suspension for $30 \mathrm{~min}$. The seedlings were replanted in the soil and then harvested at $0,0.5$, and $12 \mathrm{~h}$ and 3,5 , and 7 days after inoculation. The transgenic Arabidopsis plants were inoculation with the same method. The disease ratio was calculated as the percentage of wilting plants to the total infected plants. The disease score criterion is occurrence of newly emerging leaves of infected Arabidopsis plants showing chlorosis phenotype (Gao X. et al., 2013).

\section{Yeast Two-Hybrid Assay}

We used the pGADT7 vector containing the GAL4 AD region and the pGBKT7 vector containing GAL4 BD region for the yeast two-hybrid assay. The full-length coding sequence of GhCDKE was amplified with the listed primers (Supplementary Table S1). Enzyme-digested PCR products were cloned into the BD vector, and the coding region of the GhCDKE interacted with C-type cyclin (GhCycC) gene was cloned into the $\mathrm{AD}$ vector. About $500 \mathrm{ng}$ of plasmid DNA of each construct was co-transformed into the yeast strain AH109. The yeast two-hybrid assays were based on Matchmaker GAL4 two-hybrid systems (Clontech). The presence of the transgenes was confirmed by growth on an SD/Leu/-Trp plate. To assess protein interactions, the transformed yeasts were suspended in liquid SD/-Leu/-Trp to $\mathrm{OD}_{600}=1.0$, and $2 \mu \mathrm{L}$ of suspended yeast was dripped onto an SD/-Ade/His/-Leu/-Trp plate. Interactions were observed after 4 days of incubation at $30^{\circ} \mathrm{C}$ (Li et al., 2009).

\section{Luciferase Complementation Imaging Assay}

LCI assay was conducted as described by Zhao et al. (2014). The vectors pCAMBIA1300-cLUC and pCAMBIA1300-Nluc were kindly provided by Prof. Zhizhong Gong (College of Biological Sciences, China Agricultural University). The fulllength cDNA of GhCDKE was fused with NLUC in the pCAMBIA1300-Nluc vector, and that of GhCycC was fused with CLUC in the pCAMBIA1300-cLUC vector. The CLUCGhERF6 and GhMLP28-NLUC constructs were used as positive controls (Yang et al., 2015). Next, the plasmid constructs were transformed into A. tumefaciens GV3101. Equal amounts $(1: 1)$ of $A$. tumefaciens cultures $\left(\mathrm{OD}_{600}=1.0\right)$ containing the CLUC and NLUC constructs were mixed into the infiltration buffer (10 mM MES, pH 5.6; $200 \mathrm{mM}$ acetosyringone; and $10 \mathrm{mM} \mathrm{MgCl}_{2}$ ) and then injected into $N$. benthamiana with a needleless syringe. Tobacco plants were kept in the dark for $24 \mathrm{~h}$ and then grown under normal conditions for 2 days. Infected leaves were sprayed with $1 \mathrm{mM}$ luciferin (Sigma). A fluorescence image was obtained with a CCD camera (1300B; Roper).

\section{Virus-Induced Gene Silencing}

The TRV vectors and A. tumefaciens GV3101 were prepared as described previously (Liu et al., 2017). At the same time, the Cloroplastos alterados 1 (CLA1) gene was silenced at the initial establishment of the silent system and acted as a positive control (Supplementary Figure S3). The conserved regions of
GhCDKE and GhCLA1 (CLA1) were amplified from the cDNA of Zhongzhimian 2 with the primers shown in Supplementary Table S1 and then cloned into pTRV2 to generate pTRV2:GhCDKE and pTRV2:GhCLA1 (Gao et al., 2011). The constructs were then transformed into A. tumefaciens GV3101. The A. tumefaciens cultures were injected into the cotyledons of 2-weeks-old seedlings of Zhongzhimian 2 as described previously (Liu et al., 2017). Next, the seedlings were grown under normal conditions for 2 weeks. Next, expression of GhCDKE in infected cotton plants was detected by semi-qRT-PCR (Supplementary Figure S4). Subsequently, the GhCDKE-silenced cotton plants were challenged with $V$. dahliae by syringe inoculation as reported earlier (Liu et al., 2016).

\section{Promoter Isolation and Transient Expression}

The promoter sequence of GhPDF1.2 was obtained as previously reported (Yang et al., 2015). Primers were designed (Supplementary Table S1), and the gateway cloning system was used as described in the instruction manual (Invitrogen). The GhPDF1.2 promoter sequence was cloned into vector pGWB435, which was kindly provided by Prof. Jinsong Zhang (Institute of Genetics and Developmental Biology, Chinese Academy of Sciences), to generate pGWB435-GhPDF1.2 pro:LUC. Next, the construct was transformed into $A$. tumefaciens strain GV3101. The A. tumefaciens cultures were grown in LB medium containing $50 \mathrm{mg} / \mathrm{mL}$ rifampicin and $50 \mathrm{mg} / \mathrm{mL}$ spectinomycin. Next, A. tumefaciens cells harboring pGWB435GhPDF1.2 $2_{\text {pro }}: L U C$ were treated with infiltration buffer for $3 \mathrm{~h}$ and then injected into the leaves of 4-week wild-type (WT) and GhCDKE-silenced cotton plants. Luminescence was measured $48 \mathrm{~h}$ after infiltration using the same procedure described for the luciferase complementation imaging (LCI) assay. Relative expression of $L U C$ gene was also detected by qRT-PCR (Wang et al., 2015).

\section{RESULTS}

\section{Cloning and Sequence Analysis of GhCDKE and GhCycC}

To understand the molecular function of GhCDKE, we cloned this gene. The 1425-bp open reading frame (ORF) of GhCDKE was predicted to encode a protein of 474 amino acids with a predicted molecular mass of $53.42 \mathrm{kDa}$. CDK-related proteins in Arabidopsis were divided into seven conserved classes, CDKA-G, depending on the specific motif of their amino acid sequences, which are related to the binding of the corresponding cyclin (Vandepoele, 2002; Menges et al., 2005). Phylogenetic tree analysis showed that the protein belonged to the E-type CDKs (Supplementary Figure S1). Further, sequence alignment indicated that the protein contained the hallmark E-type CDK SPTAIRE motif involved in binding a specific cyclin, which corresponds to the SMSACRE motif in animal CDK8 amino acid sequences (Figure 1A). In addition, phylogenetic tree analysis 


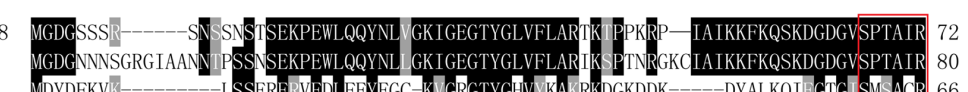
MDYDFKV

Human_CDK8

Arabidopsis_CDK8 GhCDKE

Human_CDK8

Arabidopsis_CDK8

GhCDKE

Human_CDK8

Arabidopsis_CDK8

GhCDKE

Human_CDK8

Arabidopsis_CDK8

GhCDKE

Human_CDK8

Arabidopsis_CDK8

GhCDKE

Human_CDK8

Arabidopsis_CDK8

GhCDKE

Human_CDK8

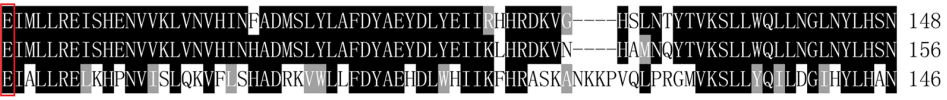

WIIHRDLKPSNILVMG DAEE G VKIADFGLARIYQAPLKPLSDNG-VVVTIWYRAPELLLGSKHYTSAVDMWAVGCIFA 227 WIIHRDLKPSNILVMGEGEEQG VKIADFGLARIYQAPLKPLS NNG VVVTIWYRAPELLLGAKHYTSAVDMWAVGCIFA 235 WV HRDLKPANILVMGEGPERRRIADMGFAR SPLKPLADLDPVVVTFWYRAPELLLGAPHYTKA D WA GCIFA 225

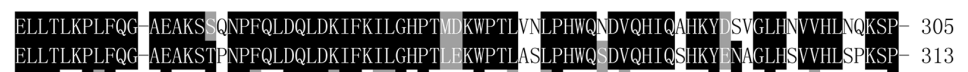
ELLTSEP FHCRQEDIKTSNP VHHDQLD IFNVMGPADKDWED KKN PEHS LUKDFRRNTYTNCSUIKYVEKHKVKPD 305
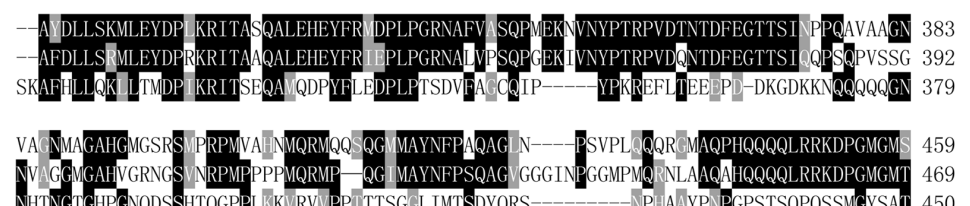

B

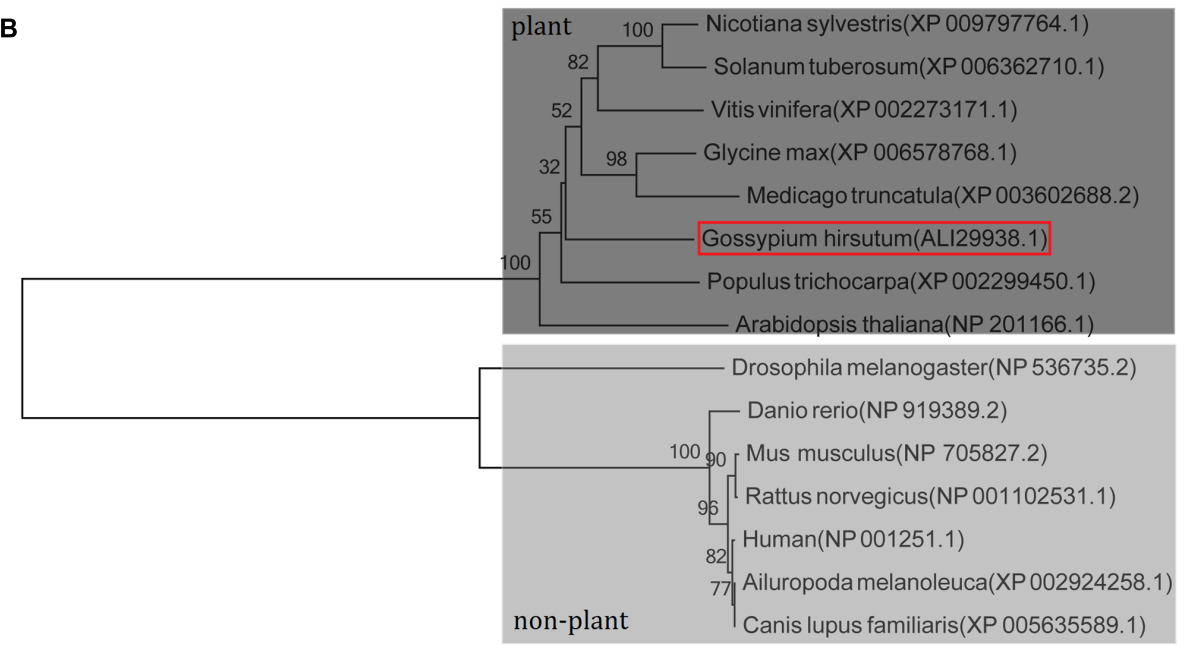

FIGURE 1 | Amino acid sequence and phylogenetic analysis of GhCDKE. (A) Amino acid sequence alignment of GhCDKE homologs in different species. The sequences in the red rectangles are the putative cyclin binding motifs. Conserved residues are shaded in black and similar in gray. Amino acid sequence alignment was performed using Clutal W. Multiple alignment file was shaded using the BoxShade program. (B) Phylogenetic tree of GhCDKE homologs. The tree is divergent into two groups, the plant group and the non-plant group. The phylogenetic tree was performed using neighbor-joining method with MEGA 5.1.

showed that the E-type CDKs of many species have diverged into two groups: the plant group and the non-plant group (Figure 1B).

Various-type CDK with its characteristic hallmark binds the corresponding cyclin. In humans, cyclin $\mathrm{C}$ partners with CDK8 (Akoulitchev et al., 2000). In this study, we cloned the corresponding partner of $\mathrm{GhCycC}$ with 252 amino acids. Phylogenetic analysis showed that the partner was in the $\mathrm{CycC}$ group (Supplementary Figure S2).

\section{Interaction of GhCDKE With GhCycC}

Previous studies have shown that CDK8 and cyclin C interacted evolutionarily with each other in yeast, humans, and Arabidopsis
(Tassan et al., 1995; Borggrefe et al., 2002; Andrau et al., 2006; Zhu et al., 2014). To elucidate whether this interaction occurred evolutionarily in cotton, we conducted a yeast twohybrid assay. For this experiment, the GhCDKE protein was fused to the GAL4-DNA binding domain, and GhCycC was fused to the GAL4-activation domain (Figure 2A). As indicated in Figure 2A, GhCDKE interacted with GhCycC. In addition, an LCI assay was conducted to assess the interaction between GhCDKE and GhCycC in plant cells. The interaction between nLuc-GhMLP28 and cLuc-GhERF6 was used as the positive control, which was confirmed in a previous study (Yang et al., 2015). As shown in Figure 2B, the Luc signal was 


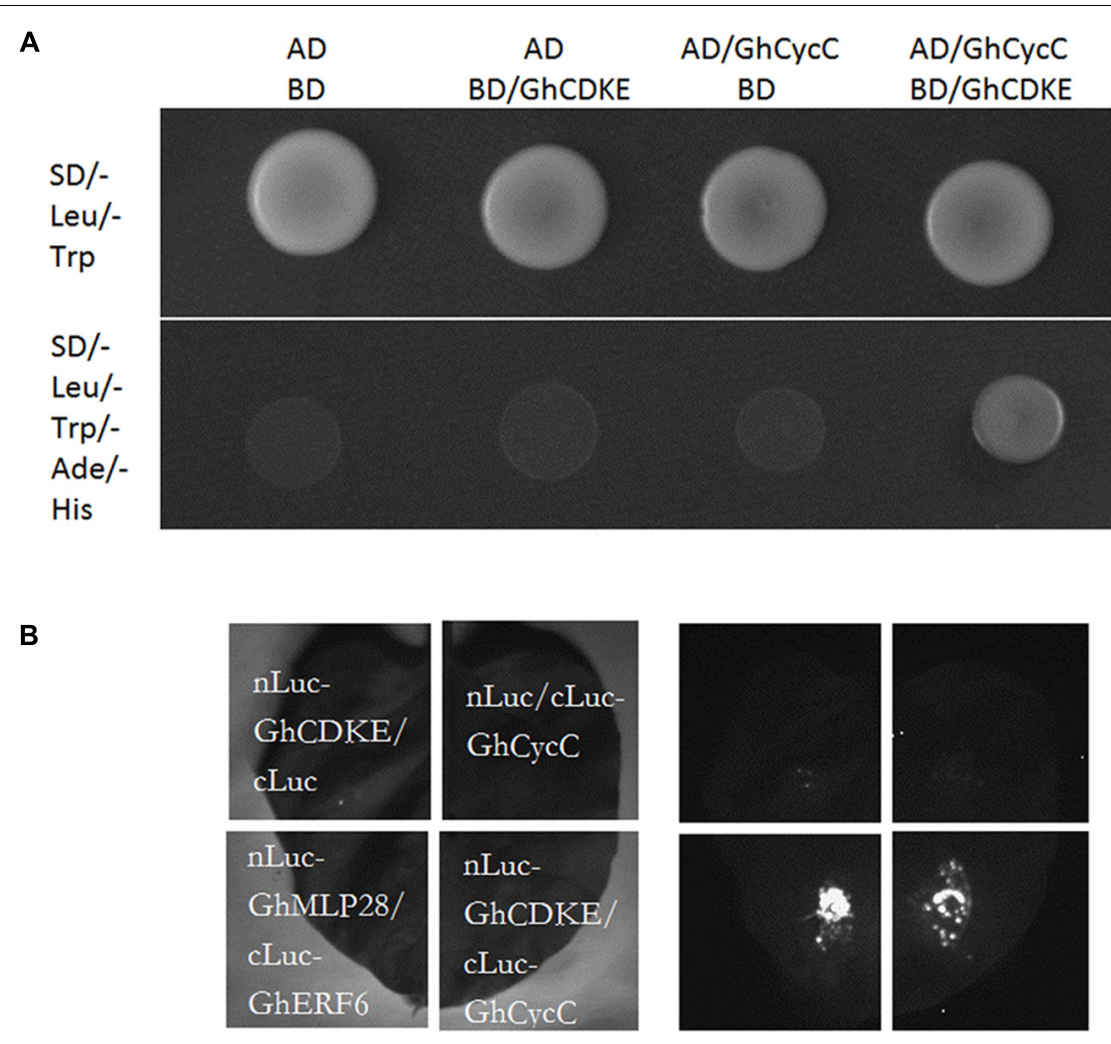

FIGURE 2 | Interaction between GhCDKE and GhCycC. (A) Interaction between GhCDKE and GhCycC in yeast. GhCDKE was fused to the GAL4-binding domain (pGBKT7), and GhCycC was fused to the GAL4-activation domain (pGADT7). (B) Luciferase complementation imaging (LCl) assay of the interaction between GhCDKE and GhCycC in tobacco leaves. Agrobacterium tumefaciens GV3101 strains harboring constructs indicated on the left panel were transferred into tobacco leaves. LCl was performed $48 \mathrm{~h}$ after co-infiltration with the same amount of $A$. tumefaciens cells. Interaction between GhMLP28 and GhERF6 was used as the positive control.

detectable only when cLuc-GhCycC/nLuc-GhCDKE or cLucGhERF6/nLuc-GhMLP28 was co-transformed into tobacco leaf cells. These results indicated that GhCDKE and GhCycC interact evolutionarily in cotton.

\section{Overexpression of GhCDKE Enhances Disease Tolerance of Arabidopsis}

Arabidopsis thaliana Mediator complex subunits, such as MED18, MED25, and SFR6/MED16, are involved in regulating plant immunity (Çevik et al., 2012; Chen et al., 2012; Wathugala et al., 2012; Lai et al., 2014; Wang et al., 2016). Thus, we hypothesized that GhCDKE is likely involved in disease response. To test whether GhCDKE is required for resistance to $V$. dahliae, we ectopically overexpressed GhCDKE in Arabidopsis. Lines 12 and 17 showed the highest expression of GhCDKE (data not shown). Using a root-dipping inoculation assay, the newly emerging leaves showed chlorosis phenotype 14 days after inoculation. The chlorosis phenotype further progresses into the older leaves. Additionally, WT plants exhibited a more sensitive and severe wilting phenotype than transgenic plants did (Figures 3A,B, lines 12 and 17), indicating that GhCDKE limits pathogen growth and disease symptoms in plants infected with Verticillium wilt.
To study the role of GhCDKE in basal resistance to $V$. dahliae, we detected related gene expression involved in the JA pathway in WT and transgenic lines after inoculation. As shown in Figure 3C, expressions of JA-regulated pathogen-responsive genes (ORA59, ERF1, PR5, PDF1.2, and CHIB) were higher in lines 12 and 17 than WT plants in response to $V$. dahliae infection, especially the expressions of PDF1.2 and ERF1 which have been proved to function in resistance to $V$. dahliae or other fungi (Brown et al., 2003; Pré et al., 2008; Zhang et al., 2012; Xing et al., 2017). In addition, expressions of JA-regulated wound-responsive genes (MYC2 and JAR1) were also activated by GhCDKE (Figure 3D) (Wassan, 2015). This result indicates that GhCDKE functions in the JA signaling pathway when plants are infected by pathogens.

\section{Knockdown of GhCDKE Expression Compromises Resistance of Cotton to V. dahliae}

To characterize the involvement of GhCDKE in cotton resistance to $V$. dahliae, we first determined its expression profile. As shown in Figure 4A, GhCDKE expression increased markedly at $0.5 \mathrm{~h}$ after inoculation of cotton seedlings with $V$. dahliae and reached the highest level at 7 days post-inoculation. To further investigate 


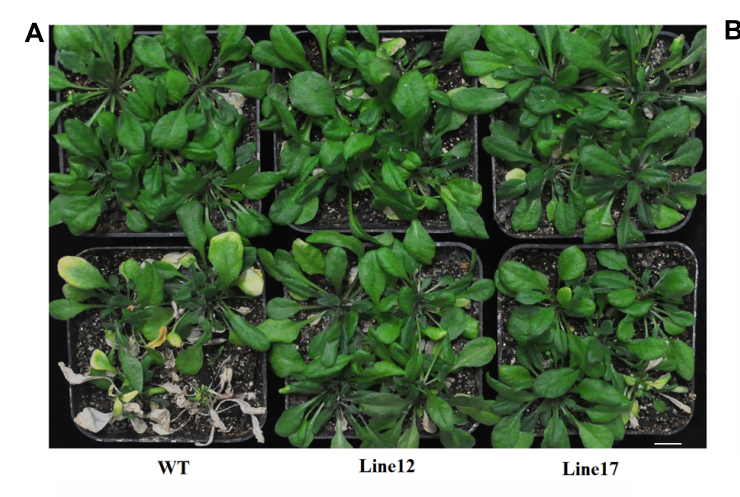

B

C

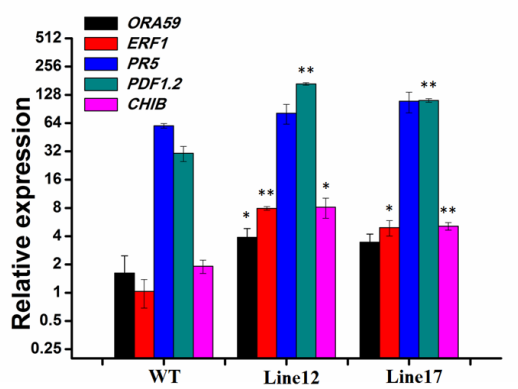

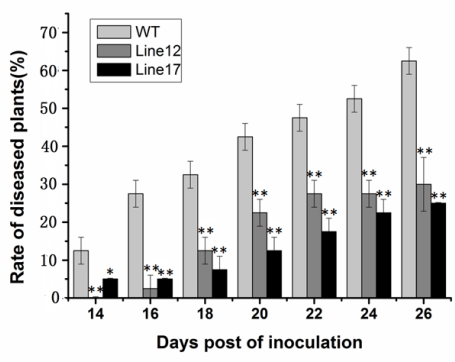

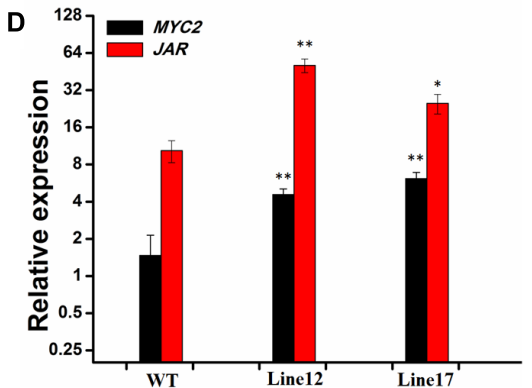

FIGURE 3 | Disease symptoms of transgenic and wild-type plants. (A) Whole plant disease phenotype of wild-type and transgenic Arabidopsis infected with V. dahliae. Photos were taken 25 days post-inoculation. Bar $=10 \mathrm{~mm}$. (B) Percentage of plants showing the Verticillium wilt phenotype at the indicated number of days after inoculation. The disease ratio was scored using 25 plants per line, and the assay was repeated three times with similar results. Expression (log2 scale) of JA-regulated pathogen-responsive genes (C) and wound-responsive genes (D) in wild-type and transgenic Arabidopsis (lines 12 and 17) plants after inoculation with $V$. dahliae. Data represent the mean $\pm S D$ of three independent biological repeats. Asterisks denote $t$-test significance compared with wild-type plants. ${ }^{*} p<0.05$ and ${ }^{* *} p<0.01$. The data were analyzed by Student's $t$-test using SPSS.

\section{A}

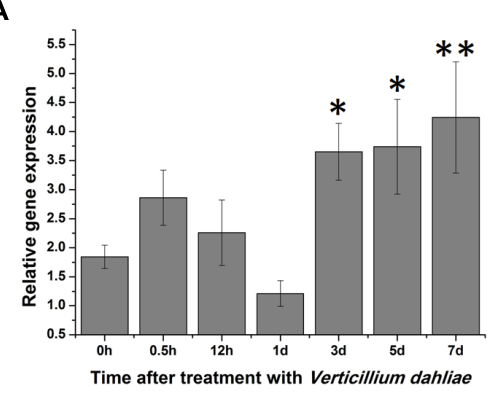

B

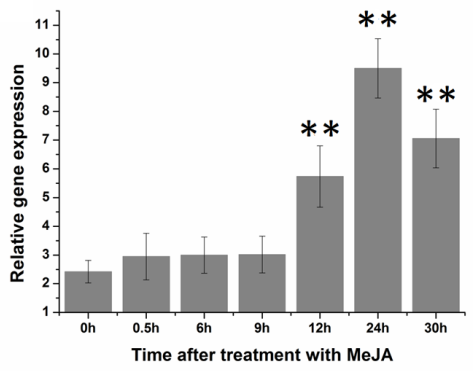

C

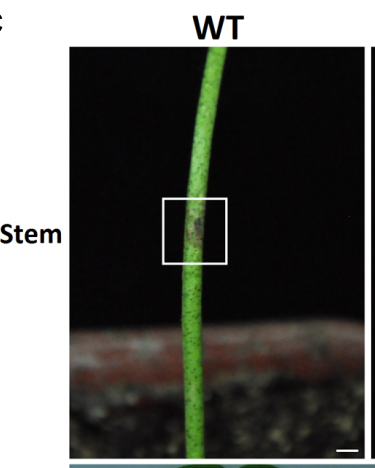

GhCDKE-silenced
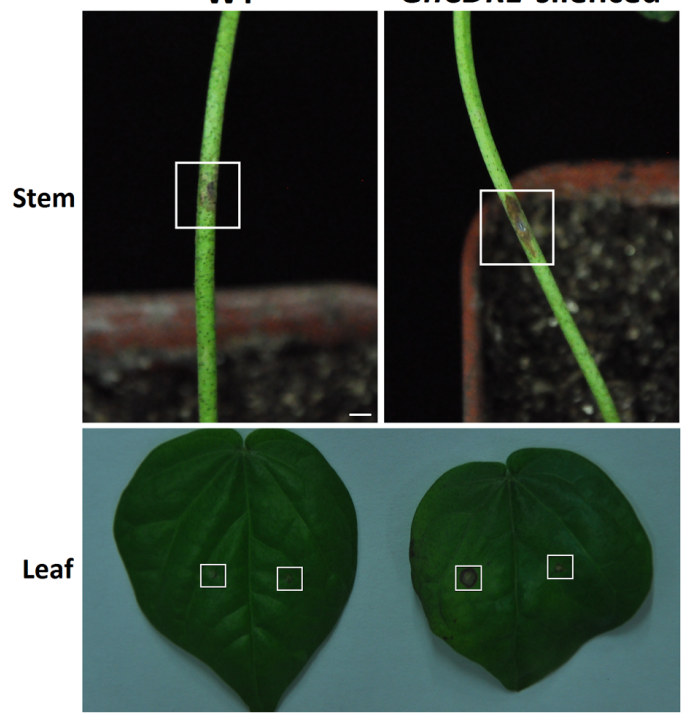

FIGURE 4 | Enhanced cotton susceptibility to V. dahliae due to GhCDKE silencing. Expression profile of the GhCDKE gene in cotton after inoculation with $V$. dahliae (A) and treatment with MeJA (B). Total RNA was extracted from 14-days-old cotton plants at the indicated times after treatment. Data are presented as mean $\pm S D$ of three biological replicates. ${ }^{*} p<0.05$ and ${ }^{* *} p<0.01$ compared to $0 \mathrm{~h}$ by one-way ANOVA followed by LSD test. (C) Disease symptoms of wild-type and GhCDKE-silenced cotton inoculated with V. dahliae. The top panel shows stem symptoms. The bottom panel shows the leaf symptoms (left, wild-type; right, GhCDKE-silenced plants), and the rectangles mark the inoculation sites (left, inoculation with $V$. dahliae; right, inoculation with water as control). Bar = $3 \mathrm{~mm}$. 


\section{A}

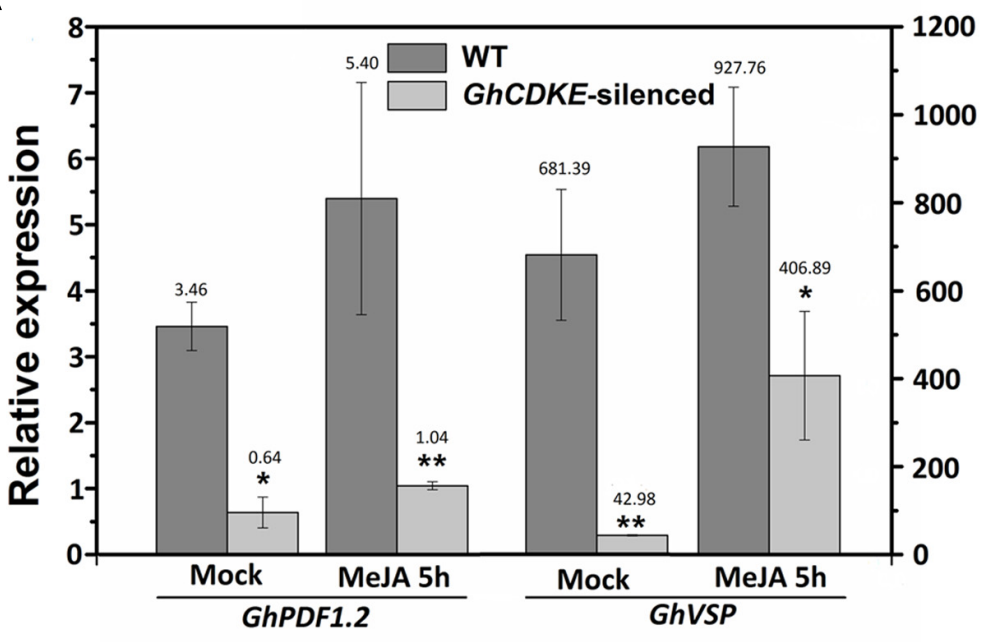

B

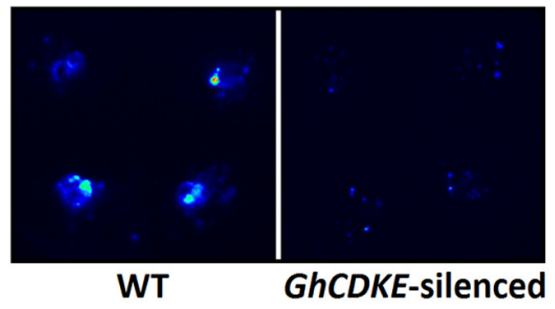

C

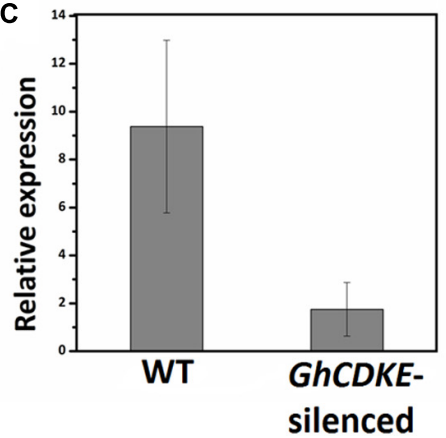

FIGURE 5 | Expression assay of GhCDKE-dependent transcription of GhPDF1.2 in cotton. (A) Quantitative RT-PCR analysis of GhPDF1.2 and GhVSP in leaves of wild-type and GhCDKE-silenced cotton lines treated with or without $100 \mu \mathrm{M}$ MeJA. The GhUBQ7 gene was used as an endogenous reference gene. Data represent the mean $\pm S D$ of three biological replicates. ${ }^{*} p<0.05$ and ${ }^{* *} p<0.01$ compared to $0 \mathrm{~h}$ by one-way ANOVA followed by LSD test. (B) Knockdown of GhCDKE reduces expression of GhPDF1.2pro:LUC. Four equal area sites were injected with the same amount of Agrobacterium tumefaciens cultures containing pGWB435-GhPDF1.2pro:LUC on every leaf. (C) Real-time polymerase chain reaction (RT-PCR) analyses of LUC transcripts from the samples in (B).

the involvement of GhCDKE in disease responses, we detected the expression of GhCDKE in cotton under treatment with methyl jasmonate (MeJA). As shown in Figure 4B, treatment of cotton seedlings with MeJA resulted in upregulation of GhCDKE expression at $12 \mathrm{~h}$ after treatment with expression peaking at $24 \mathrm{~h}$. This result supports that GhCDKE is involved in resistance to $V$. dahliae infection.

Virus-induced gene silencing is widely employed to examine the various functions of genes (Liu et al., 2002; Fu et al., 2005; Gao et al., 2011; Gao X. et al., 2013; Yang et al., 2015). To further characterize the function of $G h C D K E$ in cotton resistance to $V$. dahliae, we silenced GhCDKE by Agrobacteriummediated VIGS. Next, we inoculated the WT and GhCDKEsilenced cotton lines with spore suspensions of $V$. dahliae. As shown in Figure 4C, leaves from GhCDKE-silenced cotton had larger disease lesions than those from WT cotton at 7 days after inoculation. Disease symptoms in stems were consistent with those in leaves. We conclude that GhCDKE reduces the severity of lesions caused by $V$. dahliae infection.

\section{GhCDKE Is an Important Regulator of JA Signal Pathway}

Several subunits of the Mediator complex have been shown to be involved in hormone signaling regulation, especially the JA signaling pathway (Wathugala et al., 2012; Zhang et al., 2012; Yang et al., 2014). To determine the regulatory function of $G h C D K E$ in the JA signal pathway, we evaluated the expression of JA-responsive marker genes (GhVSP and GhPDF1.2) in WT and $G h C D K E$-silenced cotton lines. As shown in Figure $\mathbf{5 A}$, the level of GhVSP and GhPDF1.2 transcripts in GhCDKEsilenced lines was lower than that in WT lines. The transcripts were induced by JA treatment, but fewer GhVSP and GhPDF1.2 transcripts were found in GhCDKE-silenced lines. In addition, expression of $L u c$ reporter gene driven by GhPDF1.2 promoters was detected in WT and GhCDKE-silenced cotton plants. As shown in Figure 5B, promoter-reporter fusion assays revealed that GhCDKE knockdown inhibited expression GhPDF1.2 pro:Luc (for luciferase). Consistent with this result, qRT-PCR assays showed that the expression of Luc gene driven by the GhPDF1.2 


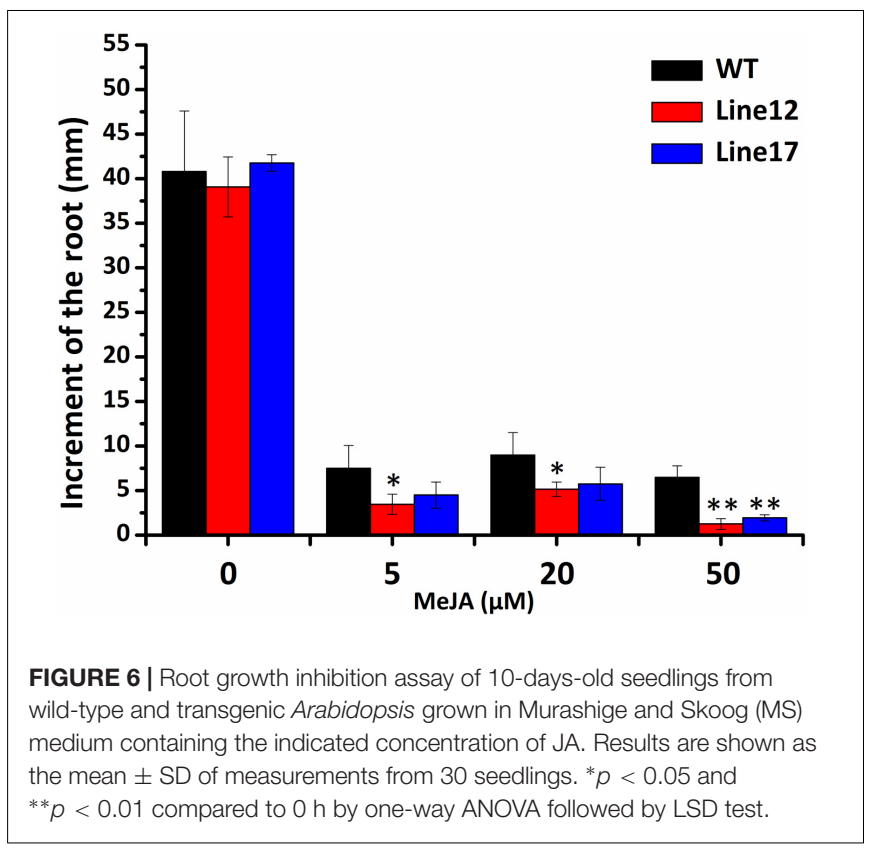

promoter was markedly reduced in GhCDKE-silenced cotton lines (Figure 5C). These results suggested that GhCDKE functions as an important regulator in JA signal pathway.

\section{The Activated JA Response Caused by GhCDKE}

Cotton cyclin-dependent kinase E regulated the expression of JA-responsive genes indicating its function in JA-signal pathway. To further characterize the role of GhCDKE in the JA signal pathway, we performed JA-induced root growth inhibition assays with overexpressed GhCDKE and WT lines. The assay showed that the transgenic lines were more sensitive than the WT lines to JA treatment in terms of root growth inhibition (Figure 6).

\section{DISCUSSION}

Upon perception by cotton cells, $V$. dahliae triggers genomewide transcriptional reprogramming, such as with hormone pathway genes (Gao W. et al., 2013). Expression regulation of resistance-related genes plays a central role in plant response processes to various pathogens (Gao et al., 2011; Gao X. et al., 2013). Although much efforts in the field of disease resistancerelated gene regulation are devoted to gene-specific transcription factors, we know relatively little about the function of the protein complexes that interact with RNA polymerase during gene transcription, such as the Mediator complex (Xu et al., 2004; Kim et al., 2008). Using molecular and genetic approaches, here we demonstrated the function of GhCDKE, a subunit of the Mediator complex. We found that first, GhCDKE physically interacted with its corresponding partner, GhCycC. Second, overexpression of GhCDKE in Arabidopsis conferred resistance to $V$. dahliae infection, and knockdown of it caused the opposite result. Third, GhCDKE was required for $V$. dahliae-induced JA-regulated pathogen-responsive gene expression.

The Mediator complex, an evolutionarily conserved multiprotein complex, functions in the transcription regulation of genes (Akoulitchev et al., 2000; Guglielmi et al., 2004; Kidd et al., 2011). It has been well studied in animal cells, especially the CDK8 subunit (Nemet et al., 2014). Mammalian CDK8 interacts with cyclin $\mathrm{C}$ to regulate the transcription of corresponding genes (Tassan et al., 1995; Akoulitchev et al., 2000; Schneider et al., 2011). The Srb10 also interacts with the Srb11 in yeast to function as a protein kinase (Borggrefe et al., 2002). Plant CDK8 was originally identified as HUA ENHANCER3 (HEN3) in Arabidopsis, which plays an important role in the specification of stamen and carpel identities (Wang and Chen, 2004). Subsequent reports have demonstrated that CDK8 interacts with CycCs to regulate plant immunity to fungal pathogens (Zhu et al., 2014). Here, we also showed that GhCDKE physically interacted with GhCycC suggesting a conserved configuration of plant Mediator kinase module and molecular mechanisms that underlie transcription regulation.

Studies have shown that the PDF1.2 expression is reduced in CDK8 mutants and regulated by WIN1/SHN1, a wax synthesis regulator that interacts with CDK8 (Zhu et al., 2014). PDF1.2 is involved in $V$. dahliae resistance (Yang et al., 2015). Thus, we used PDF1.2 to test the transcriptional regulatory function of GhCDKE in cotton. Our results showed that the transcriptional activity of PDF1.2 is dramatically decreased in GhCDKE-silenced cottons. Consistently, we observed that the expression level of PDF1.2 was obviously increased in GhCDKE-overexpressed Arabidopsis plants. Based on our results, we conclude that PDF1.2 may be a target of GhCDKE-mediated transcriptional regulation.

Previous studies have shown that JA plays a key role in plant$V$. dahliae interactions (Derksen, 2011; Tan et al., 2015). In Arabidopsis, MED16 and MED25 are involved in the regulation of the JA/ET, SA, and ABA signal pathway (Chen et al., 2012; Zhang et al., 2012, 2013). Because the Mediator complex is conserved across various eukaryotes, we hypothesized that GhCDKE likely possesses similar functions in hormone signaling regulation. MED25 is involved in the JA signaling pathway including in the defense and wound response branches. Here, we showed that GhCDKE is also involved in JA signaling pathway (Figures 3, $5,6)$. This result suggests that GhCDKE plays an essential role in relaying specific signals to RNAPII to regulate corresponding transcription. The mechanism by which GhCDKE recognizes different signals to activate corresponding gene transcription needs to be further studied.

Arabidopsis thaliana Mediator complex subunits, such as MED18, MED25, and SFR6/MED16, are involved in regulating plant immunity (Çevik et al., 2012; Chen et al., 2012; Wathugala et al., 2012; Lai et al., 2014; Wang et al., 2016). Thus, we hypothesized that GhCDKE is likely involved in disease response. Using the VIGS system, we found that silencing GhCDKE led to enhanced susceptibility to $V$. dahliae. Likewise, overexpression of GhCDKE in Arabidopsis increased resistance to $V$. dahliae. Taken together, these results indicated that GhCDKE-mediated defense may represent a broad-spectrum response in higher 
plants. Further studies regarding the mechanism of GhCDKE participation in defensive responses to various fungi need further study.

\section{AUTHOR CONTRIBUTIONS}

$\mathrm{YH}, \mathrm{FL}$, and XL conceived and designed the experiments. XL conducted most of the experiments, analyzed the data, and wrote the manuscript. NL, YS, and PW provided technical assistance to XL. NL and XG provided analysis tools. YP and DL contributed reagents and materials. All authors reviewed the manuscript.

\section{FUNDING}

This work was supported by the "Seven Crop Breeding" National Major Project (Grant No. 2016YFD0101006), the National Key Research and Development Program "Chemical Fertilizers and Pesticide Reduction Efficiency Integrated Technology Research and Development" (Grant No. 2017YFD0201902), the

\section{REFERENCES}

Akoulitchev, S., Chuikov, S., and Reinberg, D. (2000). TFIIH is negatively regulated by cdk8-containing mediator complexes. Nature 407, 102-106. doi: 10.1038/ 35024111

Andrau, J. C., Van de Pasch, L., Lijnzaad, P., Bijma, T., Koerkamp, M. G., Van de Peppel, J., et al. (2006). Genome-wide location of the coactivator mediator: binding without activation and transient Cdk8 interaction on DNA. Mol. Cell 22, 179-192. doi: 10.1016/j.molcel.2006.03.023

Borggrefe, T., Davis, R., Erdjumentbromage, H., Tempst, P., and Kornberg, R. D. (2002). A complex of the Srb8, -9, -10, and -11 transcriptional regulatory proteins from yeast. J. Biol. Chem. 277:44202. doi: 10.1074/jbc.M20719 5200

Brown, R. L., Kazan, K., McGrath, K. C., Maclean, D. J., and Manners, J. M. (2003). A role for the GCC-box in jasmonate-mediated activation of the PDF1.2 gene of Arabidopsis. Plant Physiol. 132, 1020-1032. doi: 10.1104/pp.102.017814

Caillaud, M. C., Asai, S., Rallapalli, G., Piquerez, S., Fabro, G., and Jones, J. D. (2013). A downy mildew effector attenuates salicylic acid-triggered immunity in Arabidopsis by interacting with the host Mediator complex. PLoS Biol. 11:e1001732. doi: 10.1371/journal.pbio.1001732

Canet, J. V., Dobón, A., and Tornero, P. (2012). Non-recognition-of-BTH4, an Arabidopsis mediator subunit homolog, is necessary for development and response to salicylic acid. Plant Cell 24, 4220-4235. doi: 10.1105/tpc.112. 103028

Çevik, V., Kidd, B. N., Zhang, P., Hill, C., Kiddle, S., Denby, K. J., et al. (2012). MEDIATOR25 acts as an integrative hub for the regulation of jasmonateresponsive gene expression in Arabidopsis. Plant Physiol. 160, 541-555. doi: 10.1104/pp.112.202697

Chen, R., Jiang, H., Li, L., Zhai, Q., Qi, L., Zhou, W., et al. (2012). The Arabidopsis mediator subunit MED25 differentially regulates jasmonate and abscisic acid signaling through interacting with the MYC2 and ABI5 transcription factors. Plant Cell 24, 2898-2916. doi: 10.1105/tpc.112.098277

de Jonge, R., van Esse, H. P., Maruthachalam, K., Bolton, M. D., Santhanam, P., and Saber, M. K. (2012). Tomato immune receptor Vel recognizes effector of multiple fungal pathogens uncovered by genome and RNA sequencing. Proc. Natl. Acad. Sci. U.S.A. 109, 5110-5115. doi: 10.1073/pnas.1119623109

Derksen, H. (2011). Expression of Defense Signaling Genes in the PotatoVerticillium dahliae Interaction. Master dissertation, University of Manitoba, Winnipeg, MB.

Donner, A. J., Szostek, S., Hoover, J. M., and Espinosa, J. M. (2007). CDK8 is a stimulus-specific positive coregulator of p53 target genes. Mol. Cell. 27, 121-133. doi: 10.1016/j.molcel.2007.05.026.
Genetically Modified Organism Breeding Major Project (Grant No. 2018ZX08005001-002), and the State Key Laboratory of Cotton Biology (Grant No. CB2017B03).

\section{ACKNOWLEDGMENTS}

We thank Prof. Zhizhong Gong (College of Biological Sciences, China Agricultural University) for gifts of vectors pCAMBIA1300-cLUC and pCAMBIA1300-Nluc, Prof. Jinsong Zhang (Institute of Genetics and Developmental Biology, Chinese Academy of Sciences) for gift of vector pGWB435, and Prof. Yule Liu from Tsinghua University for the VIGS constructs.

\section{SUPPLEMENTARY MATERIAL}

The Supplementary Material for this article can be found online at: https://www.frontiersin.org/articles/10.3389/fpls.2018.00642/ full\#supplementary-material

Fradin, E. F., Abd-El-Haliem, A., Masini, L., van den Berg, G. C., Joosten, M. H., and Thomma, B. P. (2011). Interfamily transfer of tomato Ve1 mediates Verticillium resistance in Arabidopsis. Plant Physiol. 156, 2255-2265. doi: 10. 1104/pp.111.180067

Fradin, E. F., and Thomma, B. P. (2006). Physiology and molecular aspects of Verticillium wilt diseases caused by V. dahliae and V. albo-atrum. Mol. Plant Pathol. 7, 71-86. doi: 10.1111/j.1364-3703.2006.00323.x

Fradin, E. F., Zhang, Z., Juarez Ayala, J. C., Castroverde, C. D., Nazar, R. N., Robb, J., et al. (2009). Genetic dissection of Verticillium wilt resistance mediated by tomato Ve1. Plant Physiol. 150, 320-332. doi: 10.1104/pp.109. 136762

Fu, D. Q., Zhu, B. Z., Zhu, H. L., Jiang, W. B., and Luo, Y. B. (2005). Virus-induced gene silencing in tomato fruit. Plant J. 43, 299-308. doi: 10.1111/j.1365-313X. 2005.02441.x

Gao, W., Long, L., Zhu, L. F., Xu, L., Gao, W. H., Sun, L. Q., et al. (2013). Proteomic and virus-induced gene silencing (VIGS) Analyses reveal that gossypol, brassinosteroids, and jasmonic acid contribute to the resistance of cotton to Verticillium dahliae. Mol. Cell. Proteomics 12, 3690-3703. doi: 10.1074/mcp.M113.031013

Gao, X., Li, F., Li, M., Kianinejad, A. S., Dever, J. K., Wheeler, T. A., et al. (2013). Cotton GhBAK1 mediates Verticillium wilt resistance and cell death. J. Integr. Plant Biol. 55, 586-596. doi: 10.1111/jipb.12064

Gao, X., Wheeler, T., Li, Z., Kenerley, C. M., He, P., and Shan, L. (2011). Silencing GhNDR1 and GhMKK2 compromises cotton resistance to Verticillium wilt. Plant J. 66, 293-305. doi: 10.1111/j.1365-313X.2011.04491.x

Guglielmi, B., van Berkum, N. L., Klapholz, B., Bijma, T., Boube, M., Boschiero, C., et al. (2004). A high resolution protein interaction map of the yeast Mediator complex. Nucleic Acids Res. 32, 5379-5391. doi: 10.1093/nar/gkh878

Hemsley, P. A., Hurst, C. H., Kaliyadasa, E., Lamb, R., Knight, M. R., De Cothi, E. A., et al. (2014). The Arabidopsis mediator complex subunits MED16, MED14, and MED2 regulate mediator and RNA polymerase II recruitment to CBF-responsive cold-regulated genes. Plant Cell 26, 465-484. doi: 10.1105/tpc. 113.117796

Kawchuk, L. M., Hachey, J., Lynch, D. R., Kulcsar, F., Rooijen, G. V., Waterer, D. R., et al. (2001). Tomato Ve disease resistance genes encode cell surfacelike receptors. Proc. Natl. Acad. Sci. U.S.A. 98, 6511-6515. doi: 10.1073/pnas. 091114198

Kidd, B. N., Cahill, D. M., Manners, J. M., Schenk, P. M., and Kazan, K. (2011). Diverse roles of the Mediator complex in plants. Semin. Cell Dev. Biol . 22, 741-748. doi: 10.1016/j.semcdb.2011.07.012

Kidd, B. N., Edgar, C. I., Kumar, K. K., Aitken, E. A., Schenk, P. M., Manners, J. M., et al. (2009). The mediator complex subunit PFT1 is a key regulator 
of jasmonate-dependent defense in Arabidopsis. Plant Cell 21, 2237-2252. doi: $10.1105 /$ tpc. 109.066910

Kim, K. C., Lai, Z., Fan, B., and Chen, Z. (2008). Arabidopsis WRKY38 and WRKY62 transcription factors interact with histone deacetylase 19 in basal defense. Plant Cell 20, 2357-2371. doi: 10.1105/tpc.107.055566

Kim, Y. J., Björklund, S., Li, Y., Sayre, M. H., and Kornberg, R. D. (1994). A multiprotein mediator of transcriptional activation and its interaction with the C-terminal repeat domain of RNA polymerase II. Cell 77, 599-608. doi: 10.1016/0092-8674(94)90221-6

Koleske, A. J., and Young, R. A. (1994). An RNA polymerase II holoenzyme responsive to activators. Nature 368, 466-469. doi: 10.1038/368466a0

Lai, Z., Schluttenhofer, C. M., Bhide, K., Shreve, J., Thimmapuram, J., Lee, S. Y., et al. (2014). MED18 interaction with distinct transcription factors regulates multiple plant functions. Nat. Commun. 5:3064. doi: 10.1038/ncomms4064

Li, Y. S., Li, X. D., Wu, Y., Li, D. N., and Li, F. (2009). Screening of Pak4 interacting protein by yeast two-hybrid system. J. China Med. Univ. 38, 7-8.

Liu, N., Ma, X., Zhou, S., Wang, P., Sun, Y., Li, X., et al. (2016). Molecular and functional characterization of a polygalacturonase-inhibiting protein from Cynanchum komarovii that confers fungal resistance in Arabidopsis. PLoS One 11:e0146959. doi: 10.1371/journal.pone.0146959

Liu, N., Zhang, X., Sun, Y., Wang, P., Li, X., Pei, Y., et al. (2017). Molecular evidence for the involvement of a polygalacturonase-inhibiting protein, GhPGIP1, in enhanced resistance to Verticillium and Fusarium wilts in cotton. Sci. Rep. 7:39840. doi: 10.1038/srep39840

Liu, Y., Schiff, M., and Dineshkumar, S. P. (2002). Virus-Induced Gene Silencing in Tomato. New York City, NY: Humana Press.

Ma, C., Jian, G., and Sun, W. (1997). Current status, problem and countermeasure on resistance breeding to verticillium wilt of cotton in China. Sci. Agric. Sin. 30, 58-64.

Menges, M., de Jager, S. M., Gruissem, W., and Murray, J. A. (2005). Global analysis of the core cell cycle regulators of Arabidopsis identifies novel genes, reveals multiple and highly specific profiles of expression and provides a coherent model for plant cell cycle control. Plant J. 41, 546-566. doi: 10.1111/j.1365313X.2004.02319.x

Nemet, J., Jelicic, B., Rubelj, I., and Sopta, M. (2014). The two faces of Cdk8, a positive/negative regulator of transcription. Biochimie 97, 22-27. doi: 10.1016/ j.biochi.2013.10.004

Pré, M., Atallah, M., Champion, A., De Vos, M., Pieterse, C. M., and Memelink, J. (2008). The AP2/ERF domain transcription factor ORA59 integrates jasmonic acid and ethylene signals in plant defense. Plant Physiol. 147, 1347-1357. doi: 10.1104/pp.108.117523

Schneider, E. V., Bottcher, J., Blaesse, M., Neumann, L., Huber, R., and Maskos, K. (2011). The structure of CDK8/CycC implicates specificity in the CDK/cyclin family and reveals interaction with a deep pocket binder. J. Mol. Biol. 412, 251-266. doi: 10.1016/j.jmb.2011.07.020

Talarczyk, A., Krzymowska, M., Borucki, W., and Hennig, J. (2002). Effect of yeast CTAl gene expression on response of tobacco plants to tobacco mosaic virus infection. Plant Physiol. 129, 1032. doi: 10.1104/pp.010960

Tan, G., Liu, K., Kang, J., Xu, K., Zhang, Y., Hu, L., et al. (2015). Transcriptome analysis of the compatible interaction of tomato with Verticillium dahliae using RNA-sequencing. Front. Plant Sci. 6:428. doi: 10.3389/fpls.2015.00428

Tassan, J. P., Jaquenoud, M., Leopold, P., Schultz, S. J., and Nigg, E. A. (1995). Identification of human cyclin-dependent kinase 8, a putative protein kinase partner for cyclin C. Proc. Natl. Acad. Sci. U.S.A. 92, 8871-8875. doi: 10.1073/ pnas.92.19.8871

Vandepoele, K. (2002). Genome-wide analysis of core cell cycle genes in Arabidopsis. Plant Cell 14, 903-916. doi: 10.1105/tpc.010445

Wang, C., Dong, X., Jin, D., Zhao, Y., Xie, S., Li, X., et al. (2015). Methyl-CpGbinding domain protein MBD7 is required for active DNA demethylation in Arabidopsis. Plant Physiol. 167, 905-914. doi: 10.1104/pp.114.252106

Wang, C., Du, X., and Mou, Z. (2016). The mediator complex subunits MED14, MED15, and MED16 are involved in defense signaling crosstalk in Arabidopsis. Front. Plant Sci. 7:1947. doi: 10.3389/fpls.2016.01947
Wang, W., and Chen, X. (2004). HUA ENHANCER3 reveals a role for a cyclindependent protein kinase in the specification of floral organ identity in Arabidopsis. Development 131, 3147-3156. doi: 10.1242/dev.01187

Wassan, G. M. (2015). Functional Analysis of Myc2-Like Gene in Cotton Responsive to Verticillium dahliae. Wuhan: Central China Agricultural University.

Wathugala, D. L., Hemsley, P. A., Moffat, C. S., Cremelie, P., Knight, M. R., and Knight, H. (2012). The Mediator subunit SFR6/MED16 controls defence gene expression mediated by salicylic acid and jasmonate responsive pathways. New Phytol. 195, 217-230. doi: 10.1111/j.1469-8137.2012.04138.x

Xing, L., Di, Z., Yang, W., Liu, J., Li, M., Wang, X., et al. (2017). Overexpression of ERF1-V from Haynaldia villosa can enhance the resistance of wheat to powdery mildew and increase the tolerance to salt and drought stresses. Front. Plant Sci. 8:1948. doi: 10.3389/fpls.2017.01948

Xu, L., Zhang, W., He, X., Liu, M., Zhang, K., Shaban, M., et al. (2014). Functional characterization of cotton genes responsive to Verticillium dahliae through bioinformatics and reverse genetics strategies. J. Exp. Bot. 65, 6679-6692. doi: $10.1093 / \mathrm{jxb} / \mathrm{eru} 393$

Xu, Y. H., Wang, J. W., Wang, S., Wang, J. Y., and Chen, X. Y. (2004). Characterization of GaWRKY1, a cotton transcription factor that regulates the sesquiterpene synthase gene $(+)-\delta$-cadinene synthase-A. Plant Physiol. 135, 507-515. doi: 10.1104/pp.104.038612

Yang, C. L., Liang, S., Wang, H. Y., Han, L. B., Wang, F. X., Cheng, H. Q., et al. (2015). Cotton major latex protein 28 functions as a positive regulator of the ethylene responsive factor 6 in defense against Verticillium dahliae. Mol. Plant 8, 399-411. doi: 10.1016/j.molp.2014.11.023

Yang, Y., Ou, B., Zhang, J., Si, W., Gu, H., Qin, G., et al. (2014). The Arabidopsis mediator subunit MED16 regulates iron homeostasis by associating with EIN3/EIL1 through subunit MED25. Plant J. 77, 838-851. doi: 10.1111/tpj. 12440

Zhang, X., Henriques, R., Lin, S. S., Niu, Q. W., and Chua, N. H. (2006). Agrobacterium-mediated transformation of Arabidopsis thaliana using the floral dip method. Nat. Protoc. 1, 641-646. doi: 10.1038/nprot.2006.97

Zhang, X., Wang, C., Zhang, Y., Sun, Y., and Mou, Z. (2012). The Arabidopsis mediator complex subunit 16 positively regulates salicylate-mediated systemic acquired resistance and jasmonate/ethylene-induced defense pathways. Plant Cell 24, 4294-4309. doi: 10.1105/tpc.112.103317

Zhang, X., Yao, J., Zhang, Y., Sun, Y., and Mou, Z. (2013). The Arabidopsis Mediator complex subunits MED14/SWP and MED16/SFR6/IEN1 differentially regulate defense gene expression in plant immune responses. Plant J. Cell Mol. Biol. 75, 484-497. doi: 10.1111/tpj.12216

Zhao, Y., Xie, S., Li, X., Wang, C., Chen, Z., Lai, J., et al. (2014). REPRESSOR OF SILENCING5 encodes a member of the small heat shock protein family and is required for DNA Demethylation in Arabidopsis. Plant Cell 26, 2660-2675. doi: $10.1105 /$ tpc. 114.126730

Zhu, H. Q., Jian, G. L., and Song, X. X. (2004). Community structure of pathogenic type of Verticillium dahliae Kleb. in cotton field. Acta Gossypii Sin. 16, 147-151.

Zhu, Y., Schluttenhoffer, C. M., Wang, P., Fu, F., Thimmapuram, J., Zhu, J. K., et al. (2014). CYCLIN-DEPENDENT KINASE8 differentially regulates plant immunity to fungal pathogens through kinase-dependent and -independent functions in Arabidopsis. Plant Cell 26, 4149-4170. doi: 10.1105/tpc.114. 128611

Conflict of Interest Statement: The authors declare that the research was conducted in the absence of any commercial or financial relationships that could be construed as a potential conflict of interest.

Copyright (C) 2018 Li, Pei, Sun, Liu, Wang, Liu, Ge, Li and Hou. This is an open-access article distributed under the terms of the Creative Commons Attribution License (CC BY). The use, distribution or reproduction in other forums is permitted, provided the original author(s) and the copyright owner are credited and that the original publication in this journal is cited, in accordance with accepted academic practice. No use, distribution or reproduction is permitted which does not comply with these terms. 